\title{
Estradiol acts during a post-pubertal sensitive period to shorten free-running circadian period in male Octodon degus
}

\author{
Daniel L. Hummer, ${ }^{1}$ Elizabeth M. Peckham ${ }^{2}$ and Theresa M. Lee ${ }^{2,3}$ \\ ${ }^{1}$ Department of Psychology, Center for Behavioral Neuroscience, Morehouse College, 830 Westview Dr. SW, Atlanta, GA 30314 , \\ USA \\ ${ }^{2}$ Department of Psychology, University of Michigan, Ann Arbor, MI, USA \\ ${ }^{3}$ Reproductive Sciences and Neuroscience Programs, University of Michigan, Ann Arbor, MI, USA
}

Keywords: adolescence, organizational effects, SCN, sensitive period, sexual dimorphism

\begin{abstract}
The free-running circadian period is approximately $30 \mathrm{~min}$ shorter in adult male than in adult female Octodon degus. The sex difference emerges after puberty, resulting from a shortened free-running circadian period in males. Castration before puberty prevents the emergence of the sex difference, but it is not a function of circulating gonadal hormones as such, because castration later in life does not affect free-running circadian period. The aim of this study was to determine whether or not the shortening of the free-running circadian period in male degus results from exposure to gonadal hormones after puberty. We hypothesized that masculinization of the circadian period results from an organizational effect of androgen exposure during a post-pubertal sensitive period. Male degus were castrated before puberty and implanted with capsules filled with dihydrotestosterone (DHT), $17 \beta$-estradiol (E2) or empty capsules at one of three ages: peri-puberty (2-7 months), post-puberty (7-12 months), or adulthood (14-19 months). Long-term exposure to DHT or E2 did not result in a shortened free-running circadian period when administered at 2-7 or 1419 months of age. However, E2 treatment from 7 to 12 months of age decreased the free-running circadian period in castrated males. This result was replicated in a subsequent experiment in which E2 treatment was limited to 8-12 months of age. E2 treatment at 7-12 months of age had no effect on the free-running circadian period in ovariectomized females. Thus, there appears to be a post-pubertal sensitive period for sexual differentiation of the circadian system of degus, during which E2 exposure decreases the free-running circadian period in males. These data demonstrate that gonadal hormones can act during adolescent development to permanently alter the circadian system.
\end{abstract}

\section{Introduction}

The effects of gonadal hormones on circadian timing are well documented (reviewed in Kriegsfeld \& Silver, 2006). Most, if not all, of these effects are assumed to result from the indirect action of gonadal hormones on afferents to the suprachiasmatic nucleus of the hypothalamus ( $\mathrm{SCN})$, as androgen and, particularly, estrogen receptors (ERs) are relatively scarce within the SCN (reviewed in Karatsoreos \& Silver, 2007). Although this assumption may hold true for many aspects of circadian timing, it does not adequately account for data demonstrating that estrogen (Morin et al., 1977; Takahashi \& Menaker, 1980; Albers, 1981) and androgens (Daan et al., 1975; Karatsoreos et al., 2007; Iwahana et al., 2008) modulate free-running circadian period, which is believed to be generated within the pacemaker itself (Pittendrigh \& Daan, 1976). Interestingly, estrogen treatment consistently shortens the free-running circadian

Correspondence: D. L. Hummer, as above.

E-mail: dhummer@morehouse.edu

Received 17 February 2012, revised 31 May 2012, accepted 20 June 2012 period of female but not male hamsters and rats. This sex-specific response of the circadian timing mechanism to estrogen in adults is the result of perinatal androgen exposure in males (Zucker et al., 1980; Albers, 1981). Thus, gonadal hormones may act directly on the SCN to permanently alter the circadian pacemaker in a sex-specific way.

The free-running circadian period differs between adult male (23.2 h) and female (23.7 h) Octodon degus (Labyak \& Lee, 1995; Lee \& Labyak, 1997). The sex difference in the degu free-running circadian period does not emerge until 12 months of age, when it shortens significantly in males (Hummer et al., 2007). The sex difference does not result from an activational effect of circulating gonadal hormones, as castration of adult males and females does not affect the free-running circadian period (Labyak \& Lee, 1995; Jechura et al., 2000). However, this sex difference does not emerge until long after degus exhibit signs of sexual maturity. Female degus first exhibit vaginal opening, followed immediately by regular estrous cyclicity, between 2 and 3 months of age (Hummer et al., 2007; Hagenauer et al., 2011; Mahoney et al., 2011). Male degus exhibit prepucial separation at approximately 2 months of age, and this is followed by 
the appearance of androgen-dependent penile spikes between 2 and 3 months of age (Hummer et al., 2007; Hagenauer et al., 2011). Postnatal exposure to gonadal hormones is necessary for the sex difference to emerge, as castration of males and females before puberty eliminates the adult sex difference in free-running circadian period (Hummer et al., 2007). We recently discovered that androgen receptor immunoreactivity increases in the $\mathrm{SCN}$ of male but not female degus after puberty, leading up to the decrease in the freerunning circadian period exhibited by males at 12 months of age (Hummer, D.L., Mahoney, M.M. \& Lee, T.M. in preparation). Consequently, we hypothesized that masculinization of the freerunning circadian period in male degus results from an organizational effect of androgens acting during a post-pubertal sensitive period. The aim of the current study was to determine: (i) which gonadal hormone is responsible for shortening the free-running circadian period in males, (ii) whether or not there is a sensitive period for hormone exposure and (iii) whether or not females are responsive to the gonadal hormone during this sensitive period.

\section{Materials and methods}

\section{Experiment 1}

Experiment 1 was conducted to determine: (i) whether or not there is a sensitive period for hormone exposure during post-pubertal development and (ii) which gonadal hormone is responsible for decreasing the free-running circadian period in males.

\section{Animals and housing}

Male degus $(n=88)$ born into an out-bred colony at the University of Michigan were housed in polypropylene cages $(42.5 \times 22 \times 19 \mathrm{~cm})$ with one or two same-sex siblings in a 12-h : 12-h light/dark (LD) environment (average light intensity, 250 lux; $20 \pm 1{ }^{\circ} \mathrm{C}$ ), except where noted below. Degus had free access to high-protein rodent chow (Prolab RMH 2000 5P06; LabDiet) and acidified water $(2.5 \times 0.000025 \% \mathrm{HCl})$ until approximately 3 months of age, after which they were fed standard rodent chow (5001 Laboratory Rodent Diet; LabDiet) and tap water ad libitum. All procedures were approved by the Animal Care and Use Committee at the University of Michigan.

\section{Castration and hormone replacement}

Males were castrated between 5 and 6 weeks of age under $5 \%$ isoflurane anesthesia (Minrad, Orchard Park, NY, USA) and weaned into same-sex groups. Castrated males were implanted subcutaneously with empty Silastic capsules (Dow Corning, Midland, MI, USA; internal diameter, 0.078 inches; outside diameter, 0.125 inches) or Silastic capsules filled to an effective length of $5 \mathrm{~mm}$ with dihydrotestosterone propionate (DHT) (Steraloids, Newport, RI, USA) or $17 \beta$-estradiol (E2) (Sigma) under isoflurane anesthesia at 2 months $(n=28), 7$ months $(n=28)$ or 14 months $(n=23)$ of age. Capsules were replaced every other month for a duration of 5 months. Thus, hormones were administered to animals at one of three ages: 27, 7-12 or 14-19 months (Fig. 1A). In order to control for the difference between the 7-12-month and 14-19-month treatment groups, in the time interval between castration and hormone treatment an additional group of males was castrated at 8 months and implanted with empty $(n=5)$ or E2-filled $(n=4)$ capsules from 14 to 19 months of age (Fig. 1A). These males were without hormones for 6 months, the same approximate length of time as the 7-12-month treatment group.

Castrated males implanted with DHT capsules developed androgendependent penile spikes (Hummer et al., 2007) indistinguishable from
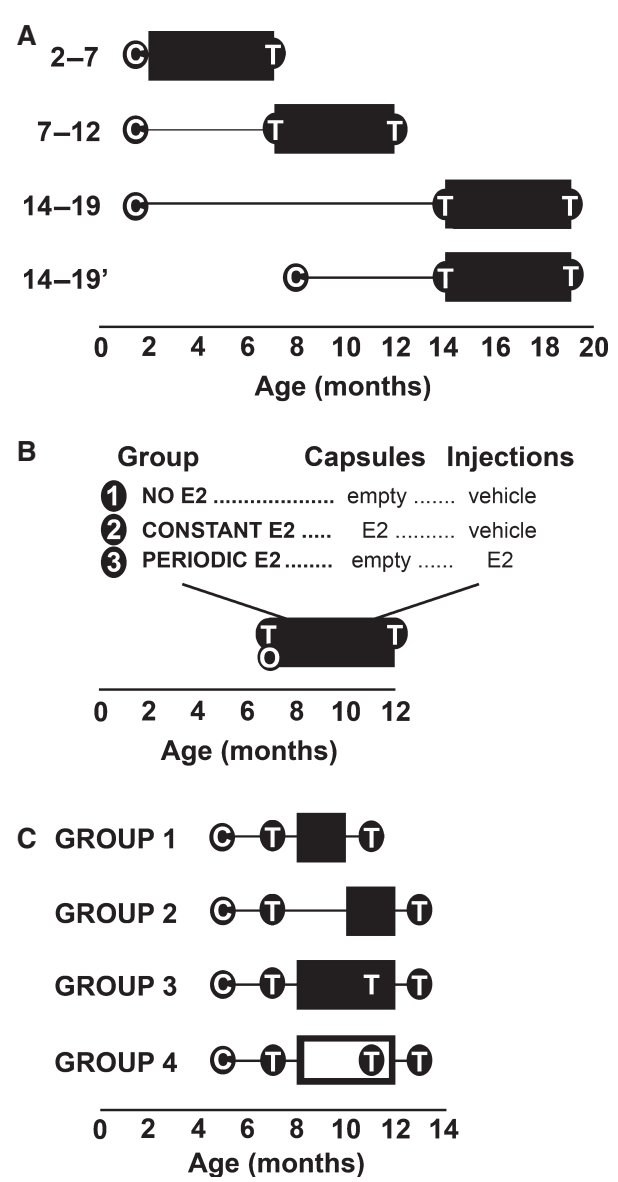

FIG. 1. Experimental design. (A) In Experiment 1, males were castrated (C) at 5-6 weeks of age and subcutaneously implanted with empty Silastic capsules or capsules filled with either DHT or E2 for 5 months (black rectangles) from 2 to 7,7 to 12 or 14 to 19 months of age. An additional group of males (14-19') was castrated at 8 months of age, and implanted with empty Silastic capsules or capsules filled with E2 from 14 to 19 months of age. The free-running circadian period was determined in constant darkness $(\mathrm{T})$ immediately prior to both the initiation and termination of hormone treatment. Note: the free-running circadian period was not determined before hormone treatment in the 2-7month treatment group. (B) In Experiment 2, females were ovariectomized (O) at 7 months, of age and subcutaneously implanted with empty Silastic capsules or capsules filled with E2 between 7 and 12 months of age (black rectangle). Females additionally received subcutaneous injections of E2 or vehicle on three consecutive days every 21 days between 7 and 12 months of age. The freerunning circadian period was determined in constant darkness $(\mathrm{T})$ immediately prior to both the initiation and termination of hormone treatment, and compared between ovariectomized females receiving no E2, constant E2, or periodic E2. (C) In Experiment 3, males were castrated (C) at 5 months of age, and subcutaneously implanted with Silastic capsules filled with E2 from 8 to 10 months (group 1), 10 to 12 months (group 2) or 8 to 12 months (group 3) of age (black rectangles). Males in group 4 received empty Silastic implants from 8 to 12 months of age (open rectangle). The free-running circadian period was determined in constant darkness (T) immediately prior to 7, 11 and/or 13 months of age.

those exhibited by gonadally intact adults. E2 capsules were smaller than those used previously (Labyak \& Lee, 1995), but sufficient to restore vaginal opening in all ovariectomized females (see Experiment 2).

\section{Testing}

Approximately 1 month prior to the initiation of hormone treatment and 1 month prior to the termination of hormone treatment, animals were separated and transferred to cages equipped with Nalgene running wheels $(9 \times 34.5 \mathrm{~cm})$ for testing. After $7-10$ days in $\mathrm{LD}$ 
$12: 12$, lights were disabled at lights out, and running wheel data were collected in constant darkness (DD) for 3-4 weeks. The freerunning circadian period was determined from running wheel data collected in DD during the 2 weeks immediately prior to the initiation of hormone treatment (Test 1) and during the 2 weeks immediately prior to the termination of hormone treatment (Test 2) (Fig. 1A). Data were not collected from the 2-7-month group prior to hormone treatment, owing to the expected negative effects of premature social isolation (Poeggel et al., 2000, 2003, 2005; Helmeke et al., 2001a,b; Ovtscharoff \& Braun, 2001).

\section{Activity data collection and analysis}

Activity data were recorded and stored in 10-min bins with VitALVIEW software and hardware (Mini-Mitter, Bend, OR, USA). The free-running circadian period was calculated by examining 24-h frequency histograms (ACTIVIEW; Mini-Mitter) after an animal had established a steady free-running circadian rhythm in DD. A regression line was drawn through daily activity onsets from at least seven consecutive days, beginning 7-14 days after release into DD. Activity onset was defined as 40 wheel revolutions in each 10-min bin for a minimum of $20 \mathrm{~min}$, following at least $4 \mathrm{~h}$ of inactivity. The free-running circadian period was calculated by an observer blind to hormone treatment, and confirmed by periodogram analysis.

\section{Statistical analyses}

One-way ANOVA was used to test the effect of HORMONE on the free-running circadian period for the 2-7-month treatment group. A $2 \times 3$ mixed effects repeated measures ANOVA was used to test the effect of TEST and HORMONE on the free-running circadian period for the 7-12-month and 14-19-month treatment groups; LITTER was included in each model as a random effect. Dunnett's test was used to make planned comparisons between animals treated with empty capsules and each of the hormone treatment groups. A $2 \times 2$ mixed effects repeated measures ANOVA was used to test the effect of TEST and HORMONE on the free-running circadian period for the 14-19month treatment group castrated at 8 months of age; LITTER was included in the model as a random effect. Data are presented as means \pm standard errors of the mean (SEMs). Group differences are considered to be statistically significant when $P<0.05$.

\section{Experiment 2}

Experiment 2 was conducted to test the prediction that the circadian system of female degus, unlike that of males, is not responsive to estrogen exposure between 7 and 12 months of age.

\section{Animals and housing}

Female degus ( $n=23$ ) born into an out-bred colony at the University of Michigan were housed and cared for as described in Experiment 1. Ovariectomized females receiving constant or periodic E2 treatment (intended to mimic the elevated E2 levels exhibited by cycling degus) between 7 and 12 months of age were compared with ovariectomized females receiving no hormone replacement.

\section{Ovariectomy and hormone replacement}

Females were ovariectomized and implanted subcutaneously with E2 or empty capsules (as described in Experiment 1) under 5\% isoflurane anesthesia at 7 months of age. In an effort to mimic the elevated E2 levels exhibited by cycling degus (Mahoney et al., 2011), one group of females implanted with empty capsules received subcutaneous injections of $5 \mu \mathrm{g}$ of $\mathrm{E} 2(0.1 \mathrm{~mL}$ in peanut oil $)$ on three consecutive days every 21 days between 7 and 12 months of age. To control for these periodic injections, all other females received subcutaneous injections of peanut oil $(0.1 \mathrm{~mL})$ on three consecutive days every 21 days between 7 and 12 months of age. This resulted in three treatment groups: (i) no E2 (empty capsule + vehicle injections, $n=7$ ), (ii) constant E2 (E2 capsule + vehicle injections, $n=8$ ) and (iii) periodic E2 (empty capsule $+\mathrm{E} 2$ injections, $n=8$ ) (Fig. 1B). E2 capsules and three consecutive days of $\mathrm{E} 2$ injections were each alone sufficient to restore vaginal opening.

\section{Testing and statistical analyses}

Approximately 1 month prior to the initiation of hormone treatment and 1 month prior to the termination of hormone treatment, animals were separated and transferred to cages equipped with Nalgene running wheels $(9 \times 34.5 \mathrm{~cm})$ for testing. After $7-10$ days in $\mathrm{LD}$ $12: 12$, lights were disabled at lights out, and running wheel data were collected in DD for 3-4 weeks. The free-running circadian period was determined from running wheel data collected in DD during the 2 weeks immediately prior to the initiation of hormone treatment (Test 1) and during the 2 weeks immediately prior to the termination of hormone treatment (Test 2) (Fig. 1B). A $2 \times 3$ mixed effects repeated measures ANOVA was used to test the overall effect of TEST and HORMONE on the free-running circadian period; LITTER was included in the model as a random effect. Dunnett's test was used to make planned comparisons between no E2 controls and each of the hormone treatment groups. Data are presented as means \pm SEMs. Group differences are considered to be statistically significant when $P<0.05$.

\section{Experiment 3}

Experiment 3 was conducted to better characterize the post-pubertal period of sensitivity to estrogen exhibited by males in Experiment 1. Specifically, the aim was to test the hypothesis that $<4$ months of estrogen exposure between 8 and 12 months of age is sufficient to permanently decrease the free-running circadian period of males.

\section{Animals and housing}

Male degus $(n=31)$ born into an out-bred colony at the University of Michigan were housed and cared for as described in Experiment 1.

\section{Castration and hormone replacement}

Male degus were castrated under 5\% isoflurane anesthesia at 5 months of age, and subsequently received one of four hormone treatments via Silastic capsule: group 1, E2 from 8 to 10 months $(n=8)$; group 2, E2 from 10 to 12 months $(n=8)$; group 3, E2 from 8 to 12 months $(n=8)$; and group 4, empty capsules from 8 to 12 months $(n=9)$. Hormone capsules were identical to those described in Experiment 1, and were replaced every 2 months (Fig. 1C).

\section{Testing and statistical analyses}

One month prior to each target age (i.e. 7, 11 or 13 months), animals were separated and transferred to cages equipped with Nalgene running wheels $(9 \times 34.5 \mathrm{~cm})$. After 7-10 days in LD $12: 12$, lights were disabled at lights out, and running wheel data were collected in DD for 3-4 weeks. The free-running circadian period was determined from running wheel data collected in DD during the 2 weeks immediately prior to 7 months (groups 1-4), 11 months (groups 1 , 3 , and 4), and 13 months (groups 2-4). In this way, all animals 
received a post-hormone test between 2 and 4 weeks after hormone capsules had been removed. Groups 3 and 4 received an additional test at 11 months of age, providing age-matched controls for group 1 (Fig. 1C). A $2 \times 3$ mixed effects repeated measures ANOVA was used to test the overall effect of TEST and HORMONE on the free-running circadian period for groups tested at 11 months (groups 1, 3, and 4) and 13 months (groups 2-4) of age. Two males in group 4 (empty capsules, 8-12 months) died prior to the 13-month test $(N=7)$. Dunnett's test was used to make planned comparisons between animals treated with empty capsules and each of the hormone treatment groups. Data are presented as means \pm SEMs. Group differences are considered statistically significant when $P<0.05$.

\section{Results}

\section{Experiment 1}

\section{Two-month to seven-month treatment}

The free-running circadian period determined at approximately 7 months of age ranged from $23.50 \pm 0.07 \mathrm{~h}$ in castrated males treated with empty capsules to $23.68 \pm 0.04 \mathrm{~h}$ in castrated males treated with DHT-filled capsules. However, there was no effect of hormone treatment on the free-running circadian period $\left(F_{2,27}=2.60\right.$, $P=0.094$; Fig. 2A).

\section{Seven-month to 12-month treatment}

The average free-running circadian period decreased in all treatment groups between 7 and 12 months of age (main effect of TEST: $\left.F_{1,42}=59.28, P<0.001\right)$. Hormone treatment had a significant effect on this change between tests (interaction between HORMONE and TEST: $\left.F_{2,42}=4.69, P=0.015\right)$. Although there were no group differences in the free-running circadian period prior to hormone treatment (Test 1), E2-treated males exhibited significantly shorter free-running circadian periods $(23.28 \pm 0.08 \mathrm{~h})$ than blank-treated males $(23.50 \pm 0.06 \mathrm{~h})$ at the conclusion of hormone treatment (Test 2; $t_{42}=-2.66, P=0.045$; Figs $2 \mathrm{~B}$ and 3 ). In contrast, the free-running circadian period did not differ between DHT-treated and blank-treated males at the conclusion of hormone treatment.

\section{Fourteen-month to 19-month treatment}

Data collected from the 14-19-month hormone treatment groups were not normally distributed, and therefore were converted to ranks prior to subsequent analysis with a mixed model ANOvA (as described by Conover \& Iman (1981). The free-running circadian period became significantly shorter, on average, between 14 and 19 months of age (main effect of TEST: $F_{1,27}=5.47, P=0.027$ ). However, there was no effect of hormone treatment on the change in free-running circadian period between tests (interaction between TEST and HORMONE: $F_{2,27}=3.07, P=0.063$; Fig. $2 \mathrm{C}$ ). Although the free-running circadian period was longer in DHT-treated $(23.65 \pm 0.03 \mathrm{~h})$ than in blanktreated males at the conclusion of hormone treatment $(23.44 \pm 0.08 \mathrm{~h})$, this difference did not reach statistical significance $\left(t_{27}=1.78, P=0.288\right)$. The fact that there was no statistically significant difference between groups at Test 2 was confirmed with non-parametric test [Kruskal-Wallis: $\chi_{2}^{2}=5.175 \quad(n=23)$, $P=0.075]$.

\section{Fourteen-month to 19-month treatment - 8-month castration}

Group averages for the free-running circadian period at 14 months of age ranged from $23.56 \pm 0.08 \mathrm{~h}$ to $23.63 \pm 0.07 \mathrm{~h}$, and appeared similar to the free-running circadian period exhibited at this same age by males castrated at 5-6 weeks of age. The free-running circadian period remained stable between 14 and 19 months of age (main effect of TEST: $F_{1,8}=0.11, P=0.750$ ) and was unaffected by hormone treatment (interaction between HORMONE and TEST: $F_{1,8}=0.21$, $P=0.657$ ) (Fig. 2D).

\section{Experiment 2}

Group averages for the free-running circadian period exhibited by ovariectomized females at 7 months of age ranged from $23.70 \pm 0.09 \mathrm{~h}$ to $23.82 \pm 0.06 \mathrm{~h}$, and remained stable between 7
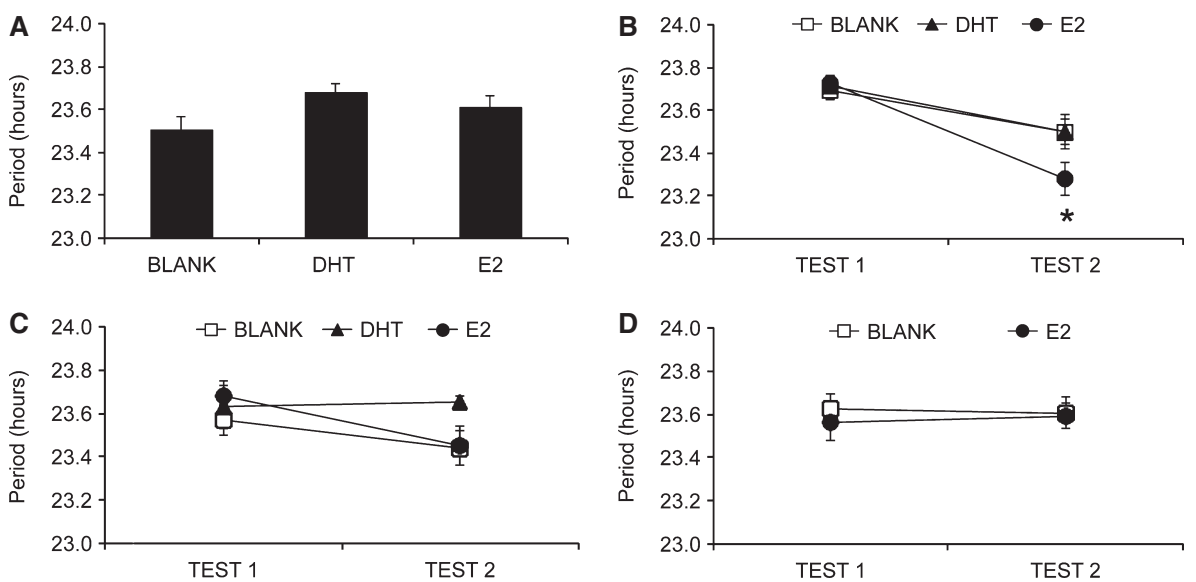

FIG. 2. (A) The effect of hormone treatment between 2 and 7 months of age on the free-running circadian period exhibited by males castrated between 5 and 6 weeks of age, tested immediately prior to the termination of hormone treatment (mean \pm SEM). (B) The effect of hormone treatment between 7 and 12 months of age on the free-running circadian period exhibited by males castrated between 5 and 6 weeks of age, tested immediately before both the initiation (Test 1 ) and termination (Test 2) of hormone treatment (mean \pm SEM, *significantly different from blank, $P<0.05$ ). (C) The effect of hormone treatment between 14 and 19 months of age on the free-running circadian period exhibited by males castrated at 5-6 weeks of age, tested immediately before both the initiation (Test 1) and termination (Test 2) of hormone treatment (mean \pm SEM). (D) The effect of hormone treatment between 14 and 19 months of age on the free-running circadian period exhibited by males castrated at 8 months of age, tested immediately before both the initiation (Test 1) and termination (Test 2) of hormone treatment (mean \pm SEM). 
and 12 months of age (main effect of TEST: $F_{1,28}=0.05, P=0.817$ ). The free-running circadian period exhibited by ovariectomized females was unaffected by hormone treatment (interaction between HORMONE and TEST: $F_{2,28}=1.36, P=0.273$ ) (Fig. 4).

\section{Experiment 3}

Seven-month vs. 11-month test (groups 1, 3, and 4)

The data collected at 7 and 11 months of age were not normally distributed, and therefore were converted to ranks prior to subsequent

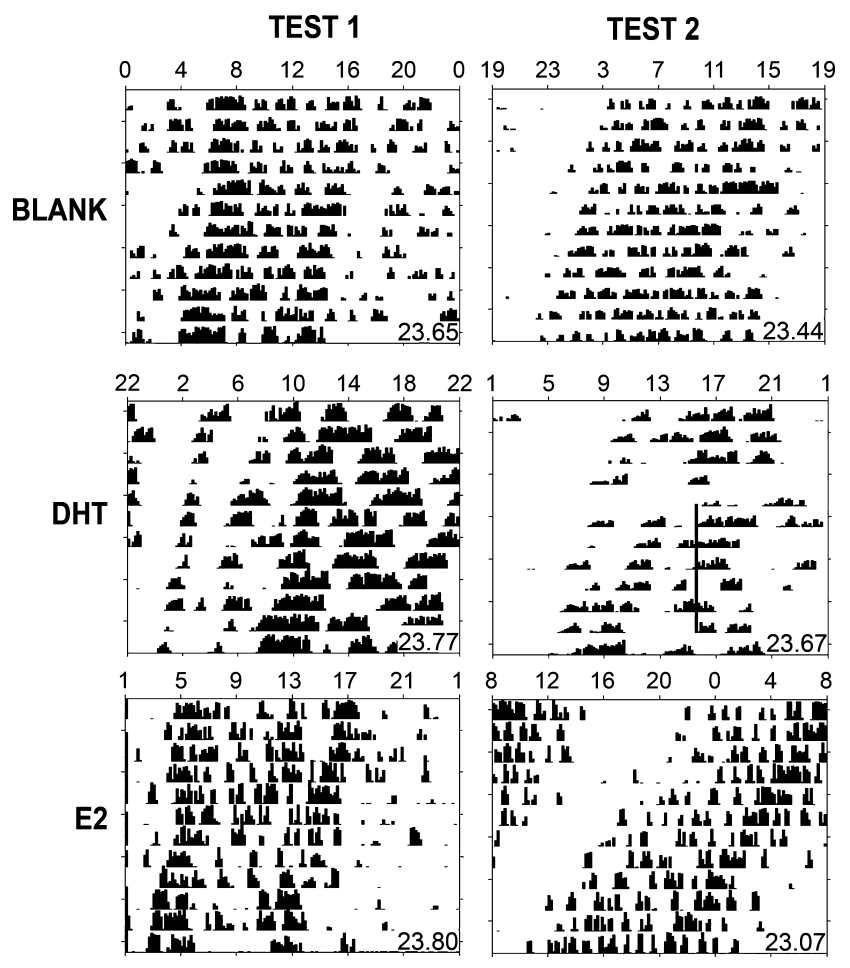

FIG. 3. Representative activity records from three castrated males receiving various hormone regimens between 7 and 12 months of age. Animals were tested in DD immediately before the initiation of hormone treatment (Test 1) and immediately before the termination of hormone treatment (Test 2) [period (h) corresponding to each rhythm depicted at lower right].

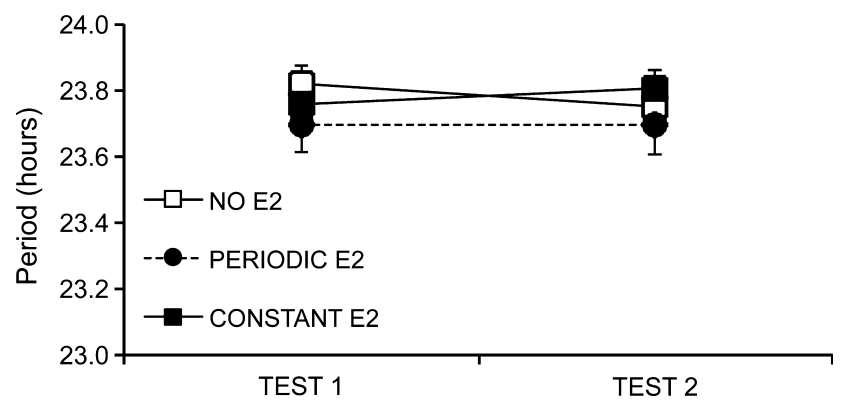

FIG. 4. The effect of hormone treatment between 7 and 12 months of age on the free-running circadian period exhibited by ovariectomized females tested immediately before both the initiation (Test 1) and termination (Test 2) of hormone treatment (mean \pm SEM; no E2, empty capsule + vehicle injections; periodic E2, empty capsule + E2 injections; constant E2, E2 capsule + vehicle injections). analysis with a mixed model ANOVA (Conover \& Iman, 1981). The group averages for the free-running circadian period exhibited by castrated males at 7 months of age ranged from $23.71 \pm 0.06 \mathrm{~h}$ to $23.81 \pm 0.05 \mathrm{~h}$, and appeared similar to the free-running circadian period exhibited by 7-month-old males in Experiment 1 $(23.69 \pm 0.04 \mathrm{~h}$ to $23.73 \pm 0.03 \mathrm{~h})$ that had been castrated at 56 weeks of age. The average free-running circadian period became significantly shorter between the 7-month and 11-month tests (main effect of TEST: $F_{1,22}=6.82, P=0.016$ ), a change that was unaffected by hormone treatment (interaction between HORMONE and TEST: $F_{2,22}=0.01, P=0.992$; Fig. 5A).

\section{Seven-month vs. 13-month test (groups 2, 3, and 4)}

Hormone treatment had a significant effect on the change in the freerunning circadian period exhibited by castrated males between 7 and 13 months of age (interaction between HORMONE and TEST: $\left.F_{2,26}=11.27, P<0.001\right)$. Although there were no group differences in the free-running circadian period prior to hormone treatment (7month test), males treated with E2 between 10 and 12 months of age exhibited significantly longer free-running circadian periods when tested after hormone treatment (13-month test; $23.75 \pm 0.04 \mathrm{~h})$ than
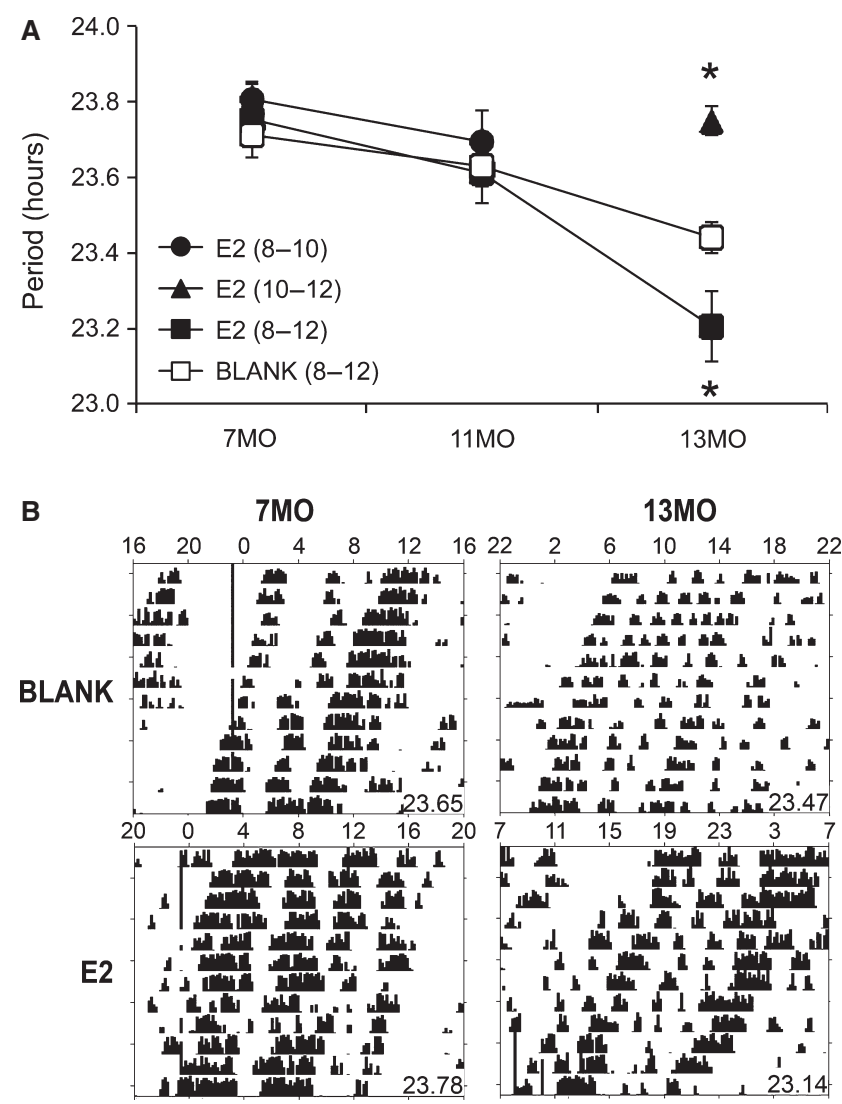

FIG. 5. (A) The effect of various hormone regimens between 8 and 12 months of age on the free-running circadian period exhibited by castrated males tested immediately prior to 7, 11 and/or 13 months of age [mean \pm SEM, *significantly different from blank, $P<0.05$; E2 $(8-10)$, E2 from 8 to 10 months of age; E2 (10-12), E2 from 10 to 12 months of age; E2 (8-12), E2 from 8 to 12 months of age; blank (8-12), empty capsules from 8 to 12 months of age]. (B) Representative activity records from two castrated males receiving either an empty Silastic capsule (blank) or Silastic capsule filled with E2 between 8 and 12 months of age. Animals were tested in DD immediately before 7 and 13 months of age [period (h) corresponding to each rhythm depicted at lower right]. 
males treated with blank capsules $\left(23.44 \pm 0.04 \mathrm{~h} ; t_{26}=6.75\right.$, $P<0.001)$. Males treated with E2 between 8 and 12 months of age exhibited significantly shorter free-running circadian periods when tested after hormone treatment (13-month test; $23.21 \pm 0.09 \mathrm{~h})$ than males treated with blank capsules $\left(t_{26}=-2.97, P=0.032\right.$; Fig. $5 \mathrm{~A}$ and $\mathrm{B})$.

\section{Discussion}

These data are consistent with the hypothesis that a sensitive period exists several months after puberty, during which the endogenous circadian timekeeping mechanism of male but not female degus is sensitive to estrogen. In Experiment 1, we demonstrated that neither DHT nor E2 is effective in changing the free-running circadian period in pre-pubertally castrated males when administered at 2-7 or 1419 months of age. E2 (but not DHT) treatment at 7-12 months, however, results in a significant decrease in the free-running circadian period. Unlike in castrated males, the free-running circadian period in ovariectomized females was not changed by exposure to E2 between 7 and 12 months of age (Experiment 2). Two months of E2 exposure, either at $8-10$ or $10-12$ months, is not sufficient to shorten the freerunning circadian period in castrated males (Experiment 3). However, castrated males treated with 4 months of E2 at 8-12 months of age exhibit a significantly shorter free-running circadian period than controls when tested at 13 months of age, after hormone capsules have been removed. These data provide evidence that the effect of E2 after puberty on the free-running circadian period is permanent, and are consistent with data demonstrating that manipulating gonadal hormones in adult males does not alter period (Jechura et al., 2000).

This sensitive period for sexual differentiation of the circadian period occurs post-pubertally, several months after male degus reach sexual maturity (Hummer et al., 2007). Similar organizational effects of gonadal hormones acting during adolescent development have been described for cognitive (Hier \& Crowley, 1982), anxiety (Primus \& Kellogg, 1990), sexual (Schulz et al., 2004), aggressive (Schulz \& Sisk, 2006) and social (Schulz et al., 2006) behaviors. However, this is the first and only report, to our knowledge, demonstrating that gonadal hormones can have an organizational effect on the circadian system during adolescent development. Yet degus are not the only species in which a sex difference in circadian rhythms emerges after puberty. A sex difference in chronotype emerges in humans between 17 and 19 years of age, with the greatest difference being observed at approximately 21 years of age (Roenneberg et al., 2004). Together, these data provide evidence that the adolescent circadian system is sensitive to long-term changes resulting from exposure to gonadal hormones.

Exposure to estrogen in vivo affects neural activity, intracellular feedback loops of clock genes and related protein products, and communication between individual pacemaker cells within the SCN. Takamata et al. (2011) recently demonstrated that the administration of E2 to ovariectomized rats results in increased c-Fos expression in the SCN during the light phase, suggesting that estrogen chronically enhances neural activity in the SCN. E2 administration dampens the Cry 2 mRNA cycle, and advances the phase of peak expression of Per2 mRNA within the SCN of ovariectomized rats (Nakamura et al., 2001, 2005). Also, Mahoney et al. (2009) demonstrated that E2 affects the daily pattern of vip gene expression in the $\mathrm{SCN}$ of female grass rats. Furthermore, Shinohara et al. $(2000,2001)$ demonstrated that E2 administration increases connexin-32 and connexin-36 mRNA expression in the SCN of ovariectomized rats, probably enhancing the coupling of single cell oscillators via gap junctions.

Although $\mathrm{ER} \alpha$ and/or $\mathrm{ER} \beta$ have been reported in the SCN of a variety of species, their quantity is limited (Pfaff \& Keiner, 1973;
Cintra et al., 1986; Sibug et al., 1991; Shughrue et al., 1997a,b; Hileman et al., 1999; Gundlah et al., 2000; Su et al., 2001; Kruijver \& Swaab, 2002; Mitra et al., 2003; Vida et al., 2008). Thus, it is widely assumed that estrogen's effects on circadian timing are the result of indirect action on ER-rich brain sites that project to the SCN (reviewed in Karatsoreos \& Silver, 2007). This may be true in mice, as E2 treatment of tissue explants from ovariectomized PER2::LUCIFERASE knockin mice does not alter the circadian period of PER2::LUCIFERASE expression in the SCN (Nakamura et al., 2008). However, free-running circadian period, believed to be generated within the pacemaker itself (Pittendrigh \& Daan, 1976), is modulated by estrogen in hamsters and rats (Morin et al., 1977; Takahashi \& Menaker, 1980; Albers, 1981). In fact, estrogen treatment consistently shortens the free-running circadian period of female but not of male hamsters and rats, the result of a permanent organizational effect of perinatal androgen exposure in males (Zucker et al., 1980; Albers, 1981). Fatehi \& Fatehi-Hassanabad (2008) have shown that in vitro application of E2 increases the frequency of spontaneous firing and depolarizes the cell membrane of SCN neurons. These data suggest that estrogen can act directly on the $\mathrm{SCN}$ of rats to influence neural activity. Future studies are needed to determine whether or not estrogen has a direct effect on clock gene expression in the SCN of non-mouse species.

Our data suggest that the sensitivity of the endogenous clock to estrogen after puberty is sexually dimorphic. An important question remains regarding how E2 manages to shorten the free-running circadian period of male but not female degus, as both males and females are presumably exposed to elevated E2 levels after puberty. That is, if sexual differentiation of the circadian timekeeping mechanism is an E2-mediated process, how do females avoid masculinization of the circadian period? The results from Experiment 2 demonstrate, first, that even constant exposure to E2 between 7 and 12 months of age (at a dose that restores vaginal opening in ovariectomized females and decreases the free-running circadian period in males) does not change the circadian period of females. These data indicate that, although E2 levels are elevated for only 23 days during the degu's 21-day estrous cycle (M.M. Mahoney, B.V. Rossi, \& T.M. Lee, unpublished data), insufficient exposure to E2 as such cannot explain the lack of response exhibited by females. The results from Experiment 2 demonstrate, second, that exposure to E2 in the absence of circulating progesterone does not change the freerunning circadian period in females. These data indicate that, unlike the well-described ability of progesterone to antagonize various activational effects of E2 on the circadian system (Richards, 1966; Axelson et al., 1979; Takahashi \& Menaker, 1980; Albers et al., 1981; Labyak \& Lee, 1995; Shinohara et al., 2001), progesterone does not act to inhibit a post-pubertal organizational effect of E2 on the circadian system of female degus. Third, the possibility remains that a period of estrogen sensitivity exists that occurs at a different age in female degus. Additional data are required to rule out this third alternative.

We hypothesize that a sex-specific developmental change in the amount and/or pattern of ER expression in the SCN of degus results in a sexually dimorphic sensitivity of the circadian clock to E2 after puberty. We would predict that the SCN of males (but not of females) will exhibit an increase in $\mathrm{ER} \alpha$ and/or $\mathrm{ER} \beta$ expression between 8 and 12 months of age. Future studies are needed to test this hypothesis.

\section{Conclusions}

Estrogen acts during a post-pubertal sensitive period in males to permanently decrease the free-running circadian period. Exposure to 
E2 between 8 and 12 months of age results in a decrease in the free-running circadian period exhibited by male Octodon degus; this change persists after hormone capsules are removed. Exposure to E2 before or after this age does not decrease the free-running circadian period, indicating that there is a post-pubertal developmental window during which the endogenous circadian timekeeping mechanism is sensitive to estrogen. Exposure to E2 during this developmental window does not alter the free-running circadian period in females, suggesting that the sensitivity of the endogenous circadian timekeeping mechanism is sexually dimorphic. We hypothesize that a sexspecific developmental change in the amount and/or pattern of ER expression in the SCN underlies post-pubertal masculinization of the endogenous circadian clock in degus.

\section{Acknowledgements}

The authors thank David Cotter and Michelle Smith for assistance with data collection, and Kathy Gimson, Julie Stewlow and Jim Donner for managing the degu colony. This research was supported by NSF IBN0212322 (T. M. Lee) and NICHD T32 HD 07048 (D. L. Hummer).

\section{Abbreviations}

$\mathrm{DD}$, constant darkness; DHT, dihydrotestosterone propionate; E2, 17 $\beta$ estradiol; ER, estrogen receptor; LD, light/dark; SCN, suprachiasmatic nucleus of the hypothalamus; SEM, standard error of the mean.

\section{References}

Albers, H.E. (1981) Gonadal hormones organize and modulate the circadian system of the rat. Am. J. Physiol., 241, R62-R66.

Albers, H.E., Gerall, A.A. \& Axelson, J.F. (1981) Effect of reproductive state on circadian periodicity in the rat. Physiol. Behav., 26, 21-25.

Axelson, J.F., Gerall, A.A. \& Albers, H.E. (1981) Effect of progesterone on the estrous activity cycle of the rat. Physiol. Behav., 26, 631-635.

Cintra, A., Fuxe, K., Harfstrand, A., Agnati, L.F., Miller, L.S., Greene, J.L. \& Gustafsson, J.-A. (1986) On the cellular localization and distribution of estrogen receptors in the rat tel- and diencephalon using monoclonal antibodies to human estrogen receptor. Neurochem. Int., 8, 587-595.

Conover, W.J. \& Iman, R.L. (1981) Rank transformations as a bridge between parametric and nonparametric statistics. Am. Stat., 35, 124-129.

Daan, S., Damassa, D., Pittendrigh, C.S. \& Smith, E.R. (1975) An effect of castration and testosterone replacement on a circadian pacemaker in mice (Mus musculus). Proc. Natl. Acad. Sci. USA, 72, 3744-3747.

Fatehi, M. \& Fatehi-Hassanabad, Z. (2008) Effects of 17beta-estradiol on neuronal cell excitability and neurotransmission in the suprachiasmatic nucleus of rat. Neuropsychopharmacol., 33, 1354-1364.

Gundlah, C., Kohama, S.G., Mirkes, S.J., Garyfallou, V.T., Urbanski, H.F. \& Bethea, C.L. (2000) Distribution of estrogen receptor beta (ERbeta) mRNA in hypothalamus, midbrain and temporal lobe of spayed macaque: continued expression with hormone replacement. Brain Res. Mol. Brain Res., 76, 191204.

Hagenauer, M.H., Ku, J.H. \& Lee, T.M. (2011) Chronotype changes during puberty depend on gonadal hormones in the slow-developing rodent, Octodon degus. Horm. Behav., 60, 37-45.

Helmeke, C., Ovtscharoff, W. Jr, Poeggel, G. \& Braun, K. (2001a) Juvenile emotional experience alters synaptic inputs on pyramidal neurons in the anterior cingulate cortex. Cereb. Cortex, 11, 717-727.

Helmeke, C., Poeggel, G. \& Braun, K. (2001b) Differential emotional experience induces elevated spine densities on basal dendrites of pyramidal neurons in the anterior cingulate cortex of Octodon degus. Neuroscience, 104, 927-931.

Hier, D.B. \& Crowley, W.F. Jr (1982) Spatial ability in androgen-deficient men. N. Engl. J. Med., 306, 1202-1205.

Hileman, S.M., Handa, R.J. \& Jackson, G.L. (1999) Distribution of estrogen receptor-beta messenger ribonucleic acid in the male sheep hypothalamus. Biol. Reprod., 60, 1279-1284.

Hummer, D.L., Jechura, T.J., Mahoney, M.M. \& Lee, T.M. (2007) Gonadal hormone effects on entrained and free-running circadian activity rhythms in the developing diurnal rodent Octodon degus. Am. J. Physiol. Regul. Integr. Comp. Physiol., 292, R586-R597.

Iwahana, E., Karatsoreos, I., Shibata, S. \& Silver, R. (2008) Gonadectomy reveals sex differences in circadian rhythms and suprachiasmatic nucleus androgen receptors in mice. Horm. Behav., 53, 422-430.

Jechura, T.J., Walsh, J.M. \& Lee, T.M. (2000) Testicular hormones modulate circadian rhythms of the diurnal rodent, Octodon degus. Horm. Behav., 38 243-249.

Karatsoreos, I.N. \& Silver, R. (2007) The neuroendocrinology of the suprachiasmatic nucleus as a conductor of body time in mammals. Endocrinology, 148, 5640-5647.

Karatsoreos, I.N., Wang, A., Sasanian, J. \& Silver, R. (2007) A role for androgens in regulating circadian behavior and the suprachiasmatic nucleus. Endocrinology, 148, 5487-5495.

Kriegsfeld, L.J. \& Silver, R. (2006) The regulation of neuroendocrine function: timing is everything. Horm. Behav., 49, 557-574.

Kruijver, F.P. \& Swaab, D.F. (2002) Sex hormone receptors are present in the human suprachiasmatic nucleus. Neuroendocrinology, 75, 296-305.

Labyak, S.E. \& Lee, T.M. (1995) Estrus- and steroid-induced changes in circadian rhythms in a diurnal rodent, Octodon degus. Physiol. Behav., 58, 573-585.

Lee, T.M. \& Labyak, S.E. (1997) Free-running rhythms and light- and darkpulse phase response curves for diurnal Octodon degus (Rodentia). Am. J. Physiol., 273, R278-R286.

Mahoney, M.M., Ramanathan, C., Hagenauer, M.H., Thompson, R.C., Smale, L. \& Lee, T. (2009) Daily rhythms and sex differences in vasoactive intestinal polypeptide, VIPR2 receptor and arginine vasopressin mRNA in the suprachiasmatic nucleus of a diurnal rodent, Arvicanthis niloticus. Eur. J. Neurosci., 30, 1537-1543.

Mahoney, M.M., Rossi, B.V., Hagenauer, M.H. \& Lee, T.M. (2011) Characterization of the estrous cycle in Octodon degus. Biol. Reprod., 84, 664-671.

Mitra, S.W., Hoskin, E., Yudkovitz, J., Pear, L., Wilkinson, H.A., Hayashi, S., Pfaff, D.W., Ogawa, S., Rohrer, S.P., Schaeffer, J.M., McEwen, B.S. \& Alves, S.E. (2003) Immunolocalization of estrogen receptor beta in the mouse brain: comparison with estrogen receptor alpha. Endocrinology, 144, 2055-2067.

Morin, L.P., Fitzgerald, K.M. \& Zucker, I. (1977) Estradiol shortens the period of hamster circadian rhythms. Science, 196, 305-307.

Nakamura, T.J., Shinohara, K., Funabashi, T. \& Kimura, F. (2001) Effect of estrogen on the expression of Cry 1 and Cry 2 mRNAs in the suprachiasmatic nucleus of female rats. Neurosci. Res., 41, 251-255.

Nakamura, T.J., Moriya, T., Inoue, S., Shimazoe, T., Watanabe, S., Ebihara, S. \& Shinohara, K. (2005) Estrogen differentially regulates expression of Per1 and Per2 genes between central and peripheral clocks and between reproductive and nonreproductive tissues in female rats. J. Neurosci. Res., 82, 622-630.

Nakamura, T.J., Sellix, M.T., Menaker, M. \& Block, G.D. (2008) Estrogen directly modulates circadian rhythms of PER2 expression in the uterus. Am. J. Physiol. Endocrinol. Metab., 295, E1025-E1031.

Ovtscharoff, W. Jr \& Braun, K. (2001) Maternal separation and social isolation modulate the postnatal development of synaptic composition in the infralimbic cortex of Octodon degus. Neuroscience, 104, 33-40.

Pfaff, D. \& Keiner, M. (1973) Atlas of estradiol-concentrating cells in the central nervous system of the female rat. J. Comp. Neurol., 151, 121-158.

Pittendrigh, C.S. \& Daan, S. (1976) The stability and lability of spontaneous frequency. J. Comp. Physiol., 106, 223-252.

Poeggel, G., Haase, C., Gulyaeva, N. \& Braun, K. (2000) Quantitative changes in reduced nicotinamide adenine dinucleotide phosphate-diaphorase-reactive neurons in the brain of Octodon degus after periodic maternal separation and early social isolation. Neuroscience, 99, 381-387.

Poeggel, G., Nowicki, L. \& Braun, K. (2003) Early social deprivation alters monoaminergic afferents in the orbital prefrontal cortex of Octodon degus. Neuroscience, 116, 617-620.

Poeggel, G., Nowicki, L. \& Braun, K. (2005) Early social environment interferes with the development of NADPH-diaphorase-reactive neurons in the rodent orbital prefrontal cortex. J. Neurobiol., 62, 42-46.

Primus, R.J. \& Kellogg, C.K. (1990) Gonadal hormones during puberty organize environment-related social interaction in the male rat. Horm. Behav., 24, 311-323.

Richards, M.P. (1966) Activity measured by running wheels and observation during the oestrous cycle, pregnancy and pseudopregnancy in the golden hamster. Anim. Behav., 14, 450-458.

Roenneberg, T., Kuehnle, T., Pramstaller, P.P., Ricken, J., Havel, M., Guth, A. \& Merrow, M. (2004) A marker for the end of adolescence. Curr. Biol., 14, R1038-R1039. 
Schulz, K.M. \& Sisk, C.L. (2006) Pubertal hormones, the adolescent brain, and the maturation of social behaviors: lessons from the Syrian hamster. Mol. Cell. Endocrinol., 254-255, 120-126.

Schulz, K.M., Richardson, H.N., Zehr, J.L., Osetek, A.J., Menard, T.A. \& Sisk, C.L. (2004) Gonadal hormones masculinize and defeminize reproductive behaviors during puberty in the male Syrian hamster. Horm. Behav., 45, 242-249.

Schulz, K.M., Menard, T.A., Smith, D.A., Albers, H.E. \& Sisk, C.L. (2006) Testicular hormone exposure during adolescence organizes flank-marking behavior and vasopressin receptor binding in the lateral septum. Horm. Behav., 50, 477-483.

Shinohara, K., Funabashi, T., Mitushima, D. \& Kimura, F. (2000) Effects of estrogen on the expression of connexin 32 and connexin43 mRNAs in the suprachiasmatic nucleus of female rats. Neurosci. Lett., 286, 107-110.

Shinohara, K., Funabashi, T., Nakamura, T.J. \& Kimura, F. (2001) Effects of estrogen and progesterone on the expression of connexin-36 mRNA in the suprachiasmatic nucleus of female rats. Neurosci. Lett., 309, 37-40.

Shughrue, P., Scrimo, P., Lane, M., Askew, R. \& Merchenthaler, I. (1997a) The distribution of estrogen receptor-beta mRNA in forebrain regions of the estrogen receptor-alpha knockout mouse. Endocrinology, 138, 5649-5652.

Shughrue, P.J., Lane, M.V. \& Merchenthaler, I. (1997b) Comparative distribution of estrogen receptor-alpha and -beta mRNA in the rat central nervous system. J. Comp. Neurol., 388, 507-525.
Sibug, R.M., Stumpf, W.E., Shughrue, P.J., Hochberg, R.B. \& Drews, U. (1991) Distribution of estrogen target sites in the 2-day-old mouse forebrain and pituitary gland during the 'critical period' of sexual differentiation. Brain Res. Dev. Brain Res., 61, 11-22.

Su, J.D., Qiu, J., Zhong, Y.P. \& Chen, Y.Z. (2001) Expression of estrogen receptor-alpha and -beta immunoreactivity in the cultured neonatal suprachiasmatic nucleus: with special attention to GABAergic neurons. NeuroReport, 12, 1955-1959.

Takahashi, J.S. \& Menaker, M. (1980) Interaction of estradiol and progesterone: effects on circadian locomotor rhythm of female golden hamsters. Am. J. Physiol., 239, R497-R504.

Takamata, A., Torii, K., Miyake, K. \& Morimoto, K. (2011) Chronic oestrogen replacement in ovariectomised rats attenuates food intake and augments cFos expression in the suprachiasmatic nucleus specifically during the light phase. Br. J. Nutr., 106, 1283-1289.

Vida, B., Hrabovszky, E., Kalamatianos, T., Coen, C.W., Liposits, Z. \& Kallo, I. (2008) Oestrogen receptor alpha and beta immunoreactive cells in the suprachiasmatic nucleus of mice: distribution, sex differences and regulation by gonadal hormones. J. Neuroendocrinol., 20, $1270-1277$.

Zucker, I., Fitzgerald, K.M. \& Morin, L.P. (1980) Sex differentiation of the circadian system in the golden hamster. Am. J. Physiol., 238, R97R101. 